\title{
Experiments on the Detection of Negative Hydrogen Ions in a Small-Size Tandem Plasma Source
}

\author{
Zh. Kiss'ovski, St. Lishev, A. Shivarova and Ts. Tsankov \\ Faculty of Physics, Sofia University, BG-1164 Sofia, Bulgaria
}

The study presents results from measurements of the negative hydrogen ion density by laser photo-detachment technique in the expanding plasma region of an inductively-driven tandem plasma source. The design of the source is, as follows. The high-frequency power deposition is in the smaller-size chamber of the discharge vessel (a quartz tube, $4.5 \mathrm{~cm}$ in a diameter and $30 \mathrm{~cm}$ of length) by an inductive coupling through a 9-turn coil. The second chamber of the discharge vessel - a metal cylinder with a radius of $11 \mathrm{~cm}$ and a length of $47 \mathrm{~cm}-$ is the expansion plasma region of the source, where the measurements have been carried out.

The measurements involve: (i) laser photodetachment technique, employed by using a Nd:YAG laser (Surelite III-10) operating at its second harmonic $(532 \mathrm{~nm})$ and a probe (with probe tip coaxial to the laser beam) for detection of the photodetached electrons, (ii) probe diagnostics in the expanding plasma region of the source (with a SMART-probe, axially movable) and, (iii) a Faraday cup (with a construction including a plasma electrode, an electron extraction electrode and a Faraday cup for collecting the negative ion current) for extraction of negative ions from the source.

The measurements are initial experiments regarding optimization of the negative ion production and extraction with respect to the operation of the magnetic filter for electron cooling positioned in the expanding plasma volume of the source. They are based on previous experience accumulated in a work on probe diagnostics in the expansion plasma region of the source operating in hydrogen (determination of the spatial variation of the electron energy distribution functions [1] and of the plasma parameters [2]), on determination of the negative ion concentration in the source involving the laser photodetachment technique [3] as well as on probe diagnostics in the expanding plasma region of the source with a magnetic filter located therein [4]. The gas pressure and the power applied for the discharge maintenance are the parameters varied in the experiments.

\section{References}

[1] N. Djermanova, Zh. Kiss'ovski, St. Lishev and Ts. Tsankov, "EEDF measurements in plasma expansion region of a high-frequency driven hydrogen discharge", J. Phys.: Conf. Series 63 (2007) 012013.

[2] Zh. Kiss'ovski, St. Kolev, A. Shivarova and Ts. Tsankov, "Expanding Plasma Region of an Inductively Driven Hydrogen Discharge", IEEE Trans. Plasma Sci. 35 (2007) 1149.

[3] Zh. Kiss'ovski, St. Kolev, A. Shivarova and Ts. Tsankov, "Laser photodetachment diagnostics of electronegative expanding plasmas", J. Phys.: Conf. Series (2008), in press.

[4] I. Djermanov, St. Kolev, St. Lishev, A. Shivarova and Ts. Tsankov, "Plasma behaviour affected by a magnetic filter", J. Phys.: Conf. Series 63 (2007) 012021.

Topic: 1. Fundamental processes and modelling

Corresponding Author: Tsanko Tsankov tsankov@phys.uni-sofia.bg

Sofia University, Faculty of Physics

Sofia, Bulgaria

Telephone: 0035928161 643, Fax: 0035929625276 